\title{
New variable chemically peculiar stars identified in the Hipparcos $\operatorname{archive}^{\star, \star \star}$
}

\author{
E. Paunzen and H.M. Maitzen \\ Institut für Astronomie der Universität Wien, Türkenschanzstr. 17, A-1180 Wien, Austria \\ e-mail: last_name@astro.ast.univie.ac.at
}

Received March 19; accepted May 13, 1998

\begin{abstract}
Since variability of chemically peculiar (CP) stars plays an important role for the astrophysical explanation of their outstanding behaviour, we have identified new variable CP stars listed in Renson's catalogue using the extensive Hipparcos Variability Annex. From the 293 objects found, 33 were excluded because they are no CP stars and/or have no period listed, half of the remaining stars are newly identified and half had been already included in the catalogue of variable CP stars by Catalano \& Renson (1997).

Most of the newly identified variability is due to an apparent magnetic field coupled with stellar rotation (oblique rotator model). The constraints of this model are fulfilled for all but three CP2 stars.

Variations of bona fide Am-Fm stars are exclusively explained by eclipses of binary systems. Furthermore eight candidates of the $\gamma$ Doradus group (pulsating Am-Fm stars) were detected.
\end{abstract}

Key words: stars: chemically peculiar — stars: early type — stars: variable

\section{Introduction}

Variability of "classical" Ap stars (= magnetic chemically peculiar stars of the upper main sequence) is a common phenomenon. Ludendorff (1906) already reported line variations in the spectrum of the "prototype" Ap star $\alpha^{2}$ CVn. The (asymmetrical) light curve of this star was first measured by Guthnick \& Prager (1914).

Send offprint requests to: E. Paunzen

* Based on data from the ESA Hipparcos astrometry satellite.

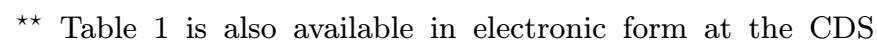
via anonymous ftp to cdsarc.u-strasbg.fr (130.79.128.5) or via http://cdsweb.u-strasbg.fr/Abstract.html
It took almost 50 years to find the physical background for this variability. Babcock (1947) and Stibbs (1950) developed the oblique rotator model for these stars in which their magnetic field is inclined at a certain angle to the rotation axis. The observed variations are therefore coupled with the apparent magnetic field as the star rotates. This theory was highly successful in explaining magnetic field variations together with photometric as well as spectral line variations. It was used to develop the technique of "Doppler-imaging" (Deutsch 1970) in order to map the abundances of elements on the stellar surface.

The second kind of variability of CP stars is due to binarity causing light variations. All type of eclipsing systems were detected and play an especially important role concerning the Am-Fm phenomenon (Budaj 1997).

We have used the extensive Hipparcos Variability Annex (HVA hereafter) in order to search for peculiar stars included in the catalogue of Ap and Am stars by Renson (1991). 293 stars were found in both catalogues (33 were excluded, see next section), half of these stars had been already known as variable, whereas 130 stars are newly identified variable CP stars (Table 1). Concerning the rotational photometric variability it has to be emphasized that the photometric band operated by Hipparcos is by no means the most suitable to detect this kind of variations: for blue magnetic (CP2) stars the amplitude decreases from the UV towards longer wavelengths, for the cooler CP2 stars the largest variability has been found to occur in the Strömgren $v$ band. On the other hand the large number of Hipparcos measurements appearing in the HVA compensate for this intrinsic disadvantage. This situation naturally calls for future ground based observations at shorter wavelengths both for confirming the periods determined and in order to establish the run of the light curve structure with wavelength. 
Table 1. a) Newly identified variable CP stars

\begin{tabular}{|c|c|c|c|c|c|c|c|c|c|c|c|}
\hline $\mathrm{HD}$ & $\mathrm{HIC}$ & RNS & Type & Min & Max & Period & Epoch & $\Delta a$ & $\Delta p$ & $v \sin i$ & Spec. \\
\hline $1826 ?$ & 1799 & 410 & ELL & 6.993 & 7.024 & 1.43237 & 8501.2256 & & 1.6 & & A3- \\
\hline $4058 ?$ & 3414 & 1120 & & 5.002 & 5.016 & 0.98211 & 8500.401 & & 1.4 & 60 & A4- \\
\hline 4161 & 3572 & 1140 & $\mathrm{EA} / \mathrm{DM}$ & 5.673 & 6.06 & 4.4673 & 8500.883 & & & 25 & $\mathrm{~A} 2-\mathrm{A} 4$ \\
\hline $16545^{*}$ & 12478 & 4120 & $\mathrm{ACV}$ & 7.319 & 7.369 & 1.61942 & 8500.6802 & & & & $\mathrm{~A} 0 \mathrm{Si}$ \\
\hline 16769 & 12821 & 4240 & $\mathrm{ACV}$ & 5.990 & 6.007 & 1.26819 & 8501.052 & & & 35 & $\mathrm{~A} 2-\mathrm{A} 6$ \\
\hline 18473 & 14049 & 4610 & $\mathrm{ACV}$ & 7.332 & 7.369 & 0.666832 & 8500.2845 & & & 75 & B9 Si \\
\hline 18597 & 13937 & 4660 & EA/DM & 8.712 & 9.237 & 2.78157 & 8500.2963 & & & & A1-F0 \\
\hline 18806 & 14087 & 4710 & $\mathrm{ACV}$ & 9.075 & 9.164 & 4.1030 & 8500.68 & & 4.5 & & $\mathrm{~A} 1 \mathrm{Cr} \mathrm{Eu} \mathrm{Sr}$ \\
\hline 21912 & 16591 & 5510 & ELL & 5.815 & 5.866 & 0.91718 & 8500.523 & & 1.2 & 100 & A3-A7 \\
\hline $23848 ?$ & 17886 & 6090 & $\mathrm{~EB}$ & 5.149 & 5.206 & 1.76532 & 8501.4890 & & -0.2 & 80 & A2- \\
\hline 24188 & 17543 & 6210 & $\mathrm{ACV}$ & 6.206 & 6.235 & 2.2301 & 8500.673 & & & $<30$ & $\mathrm{~A} 0 \mathrm{Si}$ \\
\hline 26481 & 19380 & 6720 & EA & 8.560 & 8.870 & 2.38330 & 8500.9720 & & & & $\mathrm{~A} 2-\mathrm{F} 2$ \\
\hline 26591 & 19571 & 6790 & EA & 5.840 & $>6.130$ & 3.6586 & 8502.817 & 11 & 5.5 & 45 & A1-A6 \\
\hline 26792 & 20004 & 6840 & & 6.701 & 6.743 & 3.8031 & 8501.530 & & & & $\mathrm{~B} 8 \mathrm{Sr}$ \\
\hline $26961 ?$ & 20070 & 6880 & ELL & 4.600 & 4.657 & 1.52735 & 8501.2430 & & & 95 & $\mathrm{~A} 2 \mathrm{Si}$ \\
\hline 27404 & 20262 & 7030 & $\mathrm{ACV}$ & 7.971 & 8.024 & 2.77929 & 8502.4260 & & 2.6 & & $\mathrm{~A} 0 \mathrm{Si}$ \\
\hline 32145 & 23328 & 8190 & $\mathrm{ACV}$ & 7.180 & 7.216 & 2.42082 & 8501.3551 & 37 & & & $\mathrm{~B} 8 \mathrm{Si}$ \\
\hline 34719 & 24906 & 8850 & $\mathrm{ACV}$ & 6.632 & 6.665 & 1.63988 & 8501.516 & 44 & 4.4 & & $\mathrm{~A} 0 \mathrm{Hg} \mathrm{Si} \mathrm{Cr}$ \\
\hline 294046 & 25394 & 9190 & SPB & 8.231 & 8.288 & 0.898544 & 8500.1830 & & & & B9 Si \\
\hline 39220 & 27971 & 10520 & $\mathrm{~EB}$ & 5.18 & 5.343 & 2.93326 & 8502.56 & & -0.2 & 80 & A0- \\
\hline 39575 & 27748 & 10600 & $\mathrm{ACV}$ & 7.824 & 7.867 & 3.1009 & 8501.85 & 55 & & & A0 Si Cr Eu \\
\hline 42968 & 29455 & 11470 & EA & 8.460 & 8.707 & 2.87211 & 8500.9091 & & & & $\mathrm{~A} 0-\mathrm{F} 1$ \\
\hline $44691^{*}$ & 30651 & 11840 & $\mathrm{EA} / \mathrm{DM}$ & 5.590 & 5.980 & 9.9451 & 8509.050 & & 3.1 & 30 & $\mathrm{~A} 3-\mathrm{F} 1$ \\
\hline 44953 & 30426 & 11930 & $\mathrm{ACV}$ & 6.550 & 6.579 & 5.0465 & 8501.74 & 17 & 0.9 & $<30$ & B8 He Fbl. \\
\hline 45439 & 30587 & 12040 & $\mathrm{ACV}$ & 7.830 & 7.892 & 1.10064 & 8500.6817 & 19 & 1.8 & & B9 $\mathrm{Si}$ \\
\hline 46462 & 31116 & 12430 & $\mathrm{ACV}$ & 7.466 & 7.534 & 10.3630 & 8506.734 & 25 & 0.8 & $<30$ & B9 Si \\
\hline 47144 & 31457 & 12660 & & 5.541 & 5.557 & 2.21004 & 8500.6040 & 14 & 0.7 & & B9 Si \\
\hline 47802 & 31906 & 12860 & $\mathrm{ACV}$ & 8.493 & 8.550 & 1.031227 & 8500.3646 & 30 & & & B9 Si \\
\hline 49484 & 32570 & 13390 & & 8.240 & 8.288 & 7.039 & 8500.72 & & & & B9 Si \\
\hline 49713 & 32745 & 13480 & $\mathrm{ACV}$ & 7.276 & 7.339 & 2.13503 & 8501.9352 & & 4.0 & & B9 Cr Eu Si \\
\hline 50304 & 32937 & 13774 & $\mathrm{ACV}$ & 7.556 & 7.596 & 7.884 & 8504.32 & & & & $\mathrm{~A} 0 \mathrm{Eu} \mathrm{Cr}$ \\
\hline 50341 & 33166 & 13780 & $\mathrm{ACV}$ & 8.165 & 8.222 & 2.50919 & 8500.4185 & & & & B9 Sr Cr Eu \\
\hline 52993 & 33864 & 14550 & $\mathrm{~EB}$ & 6.527 & 6.583 & 1.29644 & 8501.1043 & 29 & & 145 & B9 Si \\
\hline 56336 & 35156 & 15350 & $\mathrm{ACV}$ & 8.992 & 9.078 & 1.64029 & 8501.312 & 32 & & & B9 Si \\
\hline 56429 & 35187 & 15390 & $\mathrm{EA} / \mathrm{DM}$ & 7.968 & $>8.520$ & 4.8002 & 8502.482 & & & & $\mathrm{~A} 0-$ \\
\hline 57119 & 35407 & 15590 & $\mathrm{ACV}$ & 8.656 & 8.790 & 1.64376 & 8500.066 & & & & B9 Si \\
\hline 60559 & 36728 & 16550 & $\mathrm{~EB}$ & 6.216 & 6.243 & 1.94270 & 8501.1401 & & 0.2 & & $\mathrm{~B} 8 \mathrm{Si}$ \\
\hline 61073 & 36971 & 16700 & $\mathrm{ACV}$ & 9.118 & 9.208 & 1.96001 & 8500.185 & & & & B9 Si \\
\hline 62640 & 37692 & 17220 & $\mathrm{ACV}$ & 7.995 & 8.083 & 0.73573 & 8500.403 & 19 & & 45 & B9 Si \\
\hline 64784 & 38416 & 17760 & $\mathrm{ACV}$ & 7.468 & 7.506 & 4.0081 & 8502.38 & & & 25 & B9 Si \\
\hline $66546 ?$ & 39225 & 18393 & $\mathrm{EA}$ & 6.080 & 6.200 & 2.51465 & 8501.3100 & & 0.8 & 160 & $\mathrm{~B} 7 \mathrm{Si}$ \\
\hline $68161 ?$ & 39919 & 18840 & $\mathrm{ACV}$ & 5.622 & 5.651 & 17.028 & 8513.10 & 22 & & & $\mathrm{~B} 8$ \\
\hline 68292 & 40066 & 18860 & $\mathrm{ACV}$ & 7.465 & 7.530 & 5.7426 & 8741.69 & 35 & & $<30$ & B9 Si \\
\hline 68561 & 39791 & 19010 & $\mathrm{ACV}$ & 7.973 & 8.023 & 4.2334 & 8500.085 & 14 & & $<30$ & B9 Si \\
\hline 72175 & 41714 & 19940 & $\mathrm{ACV}$ & 9.071 & 9.127 & 4.494 & 8501.23 & & & & B9 Cr Eu Si \\
\hline 72303 & 41644 & 19990 & $\mathrm{ACV}$ & 6.387 & 6.417 & 3.7189 & 8500.830 & & & $<30$ & B9 Si \\
\hline 74067 & 42540 & 20630 & $\mathrm{ACV}$ & 5.152 & 5.196 & 3.11299 & 8502.0780 & 37 & 3.6 & 50 & A0 Cr Si \\
\hline 74888 & 42819 & 20950 & $\mathrm{ACV}$ & 6.780 & 6.833 & 1.83807 & 8500.537 & 22 & & 60 & B9 Si \\
\hline $75202 ?$ & 43071 & 21060 & & 7.736 & 7.774 & 0.290669 & 8500.1320 & & & & A3- \\
\hline 77653 & 44337 & 21970 & $\mathrm{ACV}$ & 5.191 & 5.220 & 1.48782 & 8500.0669 & 26 & 0.5 & 45 & B9 Si \\
\hline 79781 & 45693 & 22680 & $\mathrm{ACV}$ & 8.662 & 8.720 & 0.1348410 & 8500.1300 & & & & F0-F5 \\
\hline 80282 & 45548 & 22830 & $\mathrm{ACV}$ & 7.537 & 7.566 & 2.0499 & 8501.502 & 30 & 1.6 & 45 & $\mathrm{~A} 0 \mathrm{Si}$ \\
\hline 81847 & 46295 & 23260 & $\mathrm{ACV}$ & 8.280 & 8.328 & 1.41299 & 8500.8380 & 47 & 3.5 & & $\mathrm{~B} 8 \mathrm{Si}$ \\
\hline 82567 & 46452 & 23480 & $\mathrm{ACV}$ & 7.725 & 7.774 & 1.98442 & 8500.8138 & 26 & & $<30$ & B9 Si \\
\hline 82692 & 46833 & 23560 & $\mathrm{ACV}$ & 9.310 & 9.377 & 4.8910 & 8504.41 & & & & $\mathrm{~A} 0 \mathrm{Si}$ \\
\hline 85037 & 48054 & 24270 & EA & 6.560 & 6.720 & 2.72290 & 8501.2500 & & -0.1 & & A1- \\
\hline $87488 ?$ & 49375 & 25100 & $\mathrm{ACV}$ & 6.932 & 6.989 & 18.201 & 8514.843 & & & & B9 $\mathrm{Eu} \mathrm{Cr}$ \\
\hline
\end{tabular}


Table 1. b) Table 1a continued

\begin{tabular}{|c|c|c|c|c|c|c|c|c|c|c|c|}
\hline $\mathrm{HD}$ & $\mathrm{HIC}$ & $\overline{\mathrm{RNS}}$ & Type & Min & Max & Period & Epoch & $\Delta a$ & $\Delta p$ & $v \sin i$ & Spec. \\
\hline 88158 & 49642 & 25240 & $\mathrm{ACV}$ & 6.402 & 6.422 & 3.8412 & 8500.48 & & 0.9 & 60 & B8 Si \\
\hline 88603 & 49940 & 25360 & $\mathrm{ACV}$ & 7.860 & 7.917 & 1.65776 & 8501.5360 & & 0.4 & & $\mathrm{~B} 7 \mathrm{Si}$ \\
\hline 91089 & 51412 & 26200 & $\mathrm{ACV}$ & 7.449 & 7.529 & 2.15060 & 8500.558 & & & $<30$ & B9 Si \\
\hline 92106 & 51632 & 26470 & $\mathrm{ACV}$ & 7.805 & 7.837 & 25.36 & 8515.89 & & & $<30$ & $\mathrm{~A} 0 \mathrm{Sr} \mathrm{Eu} \mathrm{Cr}$ \\
\hline 93226 & 52634 & 26920 & $\mathrm{ACV}$ & 7.405 & 7.444 & 1.72901 & 8500.597 & & & & $\mathrm{~A} 0 \mathrm{Si}$ \\
\hline 95321 & 53708 & 27480 & & 9.039 & 9.090 & 0.213659 & 8500.0830 & & & & A3-A9 \\
\hline 101724 & 57067 & 29330 & $\mathrm{ACV}$ & 8.000 & 8.047 & 1.40329 & 8500.019 & 16 & 0.4 & & B9 Si \\
\hline 104810 & 58835 & 30330 & $\mathrm{ACV}$ & 7.334 & 7.399 & 2.8718 & 8501.270 & 19 & -0.3 & 120 & B8 Si \\
\hline 105509 & 59229 & 30510 & EA & 5.812 & 6.011 & 4.9664 & 8502.225 & & 2.6 & & A2-F2 \\
\hline 105770 & 59404 & 30610 & & 7.394 & 7.429 & 3.7163 & 8503.427 & 18 & & $<30$ & B9 Si \\
\hline 106112 & 59504 & 30670 & EA & 5.212 & 5.244 & 1.27095 & 8501.232 & & & 78 & A4-F3 \\
\hline 111709 & 62774 & 32420 & & 9.330 & 9.382 & 1.18567 & 8500.752 & & & & $\mathrm{~A} 4-\mathrm{A} 8$ \\
\hline 114125 & 64120 & 33030 & EA & 7.880 & 8.220 & 2.73233 & 8500.5700 & & 3.1 & & F2- \\
\hline 117057 & 65776 & 33780 & $\mathrm{ACV}$ & 8.106 & 8.168 & 1.61678 & 8501.124 & 17 & & & B9 Si \\
\hline $122314 ?$ & 68692 & 35070 & $\mathrm{EA} / \mathrm{DM}$ & 7.699 & $>8.200$ & 3.25728 & 8503.105 & & -0.4 & & A5- \\
\hline 122989 & 68979 & 35250 & $\mathrm{ACV}$ & 8.870 & 8.913 & 1.67568 & 8501.4916 & & 2.2 & & B9 Si \\
\hline 125081 & 69848 & 35740 & & 7.414 & 7.461 & 0.1539810 & 8500.0620 & & 1.2 & & F3 Sr Cr Eu \\
\hline 126198 & 70530 & 35970 & $\mathrm{ACV}$ & 7.952 & 8.039 & 4.8324 & 8500.63 & 12 & -0.4 & & B9 Si \\
\hline 127575 & 71359 & 36280 & $\mathrm{ACV}$ & 7.727 & 7.788 & 3.7263 & 8500.123 & 48 & & $<30$ & B9 Si \\
\hline 128775 & 71727 & 36640 & $\mathrm{ACV}$ & 6.561 & 6.601 & 1.63364 & 8500.4142 & & 3.1 & $<30$ & B9 Si \\
\hline $129750 ?$ & 72377 & 37000 & $\mathrm{ACV}$ & 7.082 & 7.140 & 6.987 & 8503.34 & & & $<30$ & B9 Si \\
\hline 131120 & 72800 & 37270 & SPB & 4.957 & 4.980 & 1.56895 & 8500.6542 & & -0.2 & 130 & B7 He Fbl. \\
\hline 132515 & 73479 & 37610 & EA & 8.778 & 9.094 & 1.61907 & 8500.7300 & & & & F8 Sr \\
\hline $132742 ?$ & 73473 & 37660 & $\mathrm{EA} / \mathrm{SD}$ & 4.924 & 5.933 & 2.32737 & 8502.1655 & & -0.2 & 75 & A0 \\
\hline $138764 ?$ & 76243 & 39510 & SPB & 5.111 & 5.154 & 1.25859 & 8500.180 & & 0.2 & 20 & B6 Si \\
\hline $139319 ?$ & 76196 & 39670 & $\mathrm{EA} / \mathrm{SD}$ & 7.406 & 8.963 & 2.80689 & 8500.9687 & & 0.6 & 50 & A6-F1 \\
\hline 141641 & 77657 & 40220 & $\mathrm{ACV}$ & 8.875 & 8.987 & 3.4364 & 8500.527 & 21 & 1.0 & & $\mathrm{~B} 8 \mathrm{Si}$ \\
\hline $142301^{*}$ & 77909 & 40380 & SXARI & 5.841 & 5.863 & 1.45955 & 8501.1291 & & 1.0 & 50 & B8 He Fbl.Si \\
\hline 143473 & 78533 & 40620 & $\mathrm{ACV}$ & 7.415 & 7.473 & 2.79286 & 8501.0708 & 45 & 2.2 & 30 & B9 Si \\
\hline $143939^{*}$ & 78756 & 40790 & $\mathrm{ACV}$ & 6.902 & 6.941 & 1.8489 & 8500.701 & & 4.0 & & B9 Si Cr Eu \\
\hline 145792 & 79530 & 41280 & $\mathrm{ACV}$ & 6.398 & 6.432 & 0.84780 & 8500.085 & & -0.2 & 25 & B6 He Fbl. \\
\hline 147173 & 80395 & 41580 & $\mathrm{ACV}$ & 8.706 & 8.831 & 1.85789 & 8500.659 & & & & B9 Si \\
\hline 149250 & 81554 & 42230 & $\mathrm{ACV}$ & 9.275 & 9.344 & 3.5478 & 8501.545 & & & & $\mathrm{~A} 0 \mathrm{Eu} \mathrm{Cr}$ \\
\hline $149420 ?$ & 81066 & 42290 & & 6.923 & 6.954 & 1.6972 & 8501.303 & & & 25 & A9- \\
\hline 149764 & 81477 & 42360 & $\mathrm{ACV}$ & 6.920 & 6.976 & 0.639328 & 8500.3692 & 21 & & 70 & $\mathrm{~A} 0 \mathrm{Si}$ \\
\hline 151363 & 82335 & 42820 & $\mathrm{ACV}$ & 7.781 & 7.823 & 2.57281 & 8502.3182 & & & 105 & B9 Si \\
\hline 152564 & 83150 & 43220 & $\mathrm{ACV}$ & 5.746 & 5.769 & 2.1637 & 8500.527 & & 0.9 & 75 & A0 Si \\
\hline 154856 & 84025 & 43750 & $\mathrm{ACV}$ & 8.767 & 8.824 & 1.9525 & 8501.486 & & & & $\mathrm{~B} 8 \mathrm{Si}$ \\
\hline 156049 & 84686 & 44020 & $\mathrm{ACV}$ & 8.211 & 8.306 & 1.80495 & 8500.6500 & & & & B9 Cr Eu \\
\hline 159829 & 86712 & 44960 & EA & 9.648 & 10.022 & 4.2799 & 8503.210 & & & & $\mathrm{~A} 2-\mathrm{A} 8$ \\
\hline 161841 & 87257 & 45680 & $\mathrm{ACV}$ & 7.516 & 7.564 & 3.21048 & 8500.738 & 14 & -0.2 & & B9 Si \\
\hline 162613 & 87580 & 45910 & $\mathrm{ACV}$ & 7.950 & 8.008 & 3.5832 & 8501.123 & & & & $\mathrm{~A} 0 \mathrm{Si}$ \\
\hline $165814 ?$ & 88905 & 46706 & $\mathrm{~EB}$ & 6.686 & 7.148 & 2.24810 & 8502.2030 & & -0.1 & & B9 Si \\
\hline 166427 & 89225 & 46840 & $\mathrm{ACV}$ & 8.096 & 8.145 & 1.96607 & 8500.4720 & & & 35 & B9 Si Sr \\
\hline $167356 ?$ & 89470 & 47035 & $\mathrm{ACV}$ & 6.091 & 6.123 & 2.3595 & 8501.138 & & 2.6 & 25 & $\mathrm{~A} 1 \mathrm{Si}$ \\
\hline $167858 ?$ & 89601 & 47080 & & 6.660 & 6.747 & 1.30700 & 8500.193 & & -0.3 & 13 & F0 \\
\hline $168403 ?$ & 89955 & 47190 & $\mathrm{ACV}$ & 6.802 & 6.851 & 4.7992 & 8501.584 & & & & A0 Eu Cr Sr \\
\hline 169952 & 90293 & 47600 & $\mathrm{ACV}$ & 7.321 & 7.349 & 2.1223 & 8500.673 & & 1.5 & & B9 Si \\
\hline 171184 & 91001 & 47930 & $\mathrm{ACV}$ & 7.928 & 7.995 & 2.79926 & 8500.3360 & & & & $\mathrm{~A} 0 \mathrm{Si}$ \\
\hline 171263 & 90990 & 47960 & $\mathrm{ACV}$ & 7.778 & 7.837 & 3.9980 & 8503.131 & & & & B8 Si \\
\hline 171782 & 91224 & 48130 & $\mathrm{ACV}$ & 7.832 & 7.883 & 4.466 & 8500.69 & & & & B9 Si Cr Eu \\
\hline $174638 ?$ & 92420 & 48890 & $\mathrm{~EB}$ & 3.373 & 4.317 & 12.940 & 8512.086 & & 4.0 & 120 & $\mathrm{~B} 8 \mathrm{He}$ \\
\hline $179213 ?$ & 94693 & 49790 & $\mathrm{~EB}$ & 9.067 & 9.253 & 2.50364 & 8501.3024 & & & & A3-A9 \\
\hline $182255 \backslash$ & 95260 & 50370 & SPB & 5.144 & 5.182 & 1.26239 & 8500.727 & & -0.4 & 40 & B6 He Fbl. \\
\hline 184242 & 96011 & 50840 & EA & 6.938 & $>7.180$ & 1.86742 & 8501.1262 & & & & A3-A9 \\
\hline $187418 ?$ & 97756 & 51680 & $\mathrm{~EB}$ & 8.292 & 8.750 & 1.61311 & 8500.44 & & 2.0 & & $\mathrm{~A} 2-\mathrm{A} 7$ \\
\hline
\end{tabular}


Table 1. c) Table 1b continued

\begin{tabular}{lrrrrrllllll}
\hline HD & HIC & RNS & Type & Min & Max & Period & Epoch & $\Delta a$ & $\Delta p$ & $v \sin i$ & Spec. \\
\hline $190786 ?$ & 98955 & 53060 & EA/DM & 8.566 & $>9.320$ & 2.34698 & 8500.6622 & & & 40 & A1-A3 \\
191439 & 99615 & 53340 & ACV & 8.888 & 8.927 & 1.62718 & 8500.1028 & 46 & & B9 Cr Eu Sr \\
193637 & 100258 & 54040 & EA/DM & 8.39 & 9.030 & 4.00519 & 8500.58 & & 2.5 & A5-F2 \\
196270 & 101569 & 54710 & ACV & 8.173 & 8.221 & 1.30217 & 8500.3993 & 1.7 & B9 Si \\
197018 & 101949 & 54940 & ACV & 6.020 & 6.038 & 5.960 & 8501.58 & 0.3 & B7 Mn \\
199532 & 104043 & 55600 & EB & 5.221 & 5.260 & 2.87700 & 8500.3616 & 1.9 & 85 & F2- \\
$204038 ?$ & 105739 & 56830 & EW/KE & 8.321 & 8.720 & 0.785848 & 8500.5030 & 0.2 & A3-F0 \\
205938 & 106604 & 57340 & ACV & 6.415 & 6.467 & 8.340 & 8506.79 & & & B9 Si \\
206155 & 106981 & 57410 & EA/DM & 6.997 & 7.607 & 2.62817 & 8502.2388 & & 40 & A2-A5 \\
206653 & 107525 & 57500 & ACV & 7.161 & 7.205 & 1.78706 & 8500.670 & 23 & & $<30$ & B9 Si \\
$207098 *$ & 107556 & 57630 & EB & 2.920 & $>3.070$ & 1.022767 & 8500.8500 & & 0.6 & 90 & A5-F4 DD \\
$210071 *$ & 109124 & 58470 & E & 6.332 & 6.401 & 1.43246 & 8500.9310 & 0.6 & 75 & B9 Si Cr Hg \\
213871 & 111360 & 59320 & ACV & 7.344 & 7.395 & 1.9505 & 8500.925 & & B9 Si \\
219815 & 115065 & 60340 & EB & 6.014 & 6.171 & 3.21952 & 8500.732 & & 80 A5-F2 \\
220147 & 115267 & 60450 & ACV & 8.120 & 8.175 & 10.990 & 8501.978 & & & B9 Cr Si Eu \\
$220885 ?$ & 115755 & 60550 & ACV & 5.733 & 5.770 & 1.47946 & 8500.055 & & 1.1 & 55 & B9 Mn \\
$223967 ?$ & 117853 & 61360 & ACV & 7.029 & 7.063 & 1.27712 & 8500.643 & & & B9 Si \\
\hline
\end{tabular}

Notes to Table 1:

Col. 1: HD number with probability mark according to Renson (1991).

Col. 2: HIC number.

Col. 3: Renson number.

Col. 4: type of variability, abbreviations as in the HVA.

Cols. 5, 6: Min and Max of the variations.

Col. 7: observed period in days.

Col. 8: Epoch as in the HVA.

Cols. 9, 10: $\Delta a$ in mmag and $\Delta p$.

Col. 11: $v \sin i$ value from the literature.

Col. 12: spectral type according to Renson (1991).

\section{Program stars}

The catalogue of Ap and Am stars by Renson (1991) was used to search for entries in the HVA. 293 objects were found in the first part (periodic variables) whereas 106 objects are included in the second part (unsolved variables). The latter will be discussed in a forthcoming paper. From these 293 objects, we excluded 33 because:

- Twelve are well known $\delta$ Scuti or $\delta$ Delphini stars and no "classical" CP stars, these are: HD 2628, HD 67523, HD 67911, HD 93137, HD 101696, HD 106384, HD 115604, HD 172748, HD 185139, HD 197461, HD 201707 and HD 213534.

- Eighteen have no period due to poor phase coverage or other reasons, these are: HD 5303, HD 30050, HD 34364, HD 36412, HD 40183, HD 82829, HD 89143, HD 113158, HD 128661, HD 156965, HD 161321, HD 178001, HD 185257, HD 187474, HD 205234, HD 209147, HD 215661 and HD 216429.

- Three are very probably no CP stars since the exhibit a non-peculiar $\Delta a$ index despite their B-type characteristics, these are: HD 24587 (B6; $\Delta a=-1)$, HD 38602 (B9 Si; $\Delta a=-2$ ) and HD 66546 (B7 Si; $\Delta a=-6$ ).

From the remaining 260 program stars, half have already been included in the catalogue of observed periods of Ap

and Bp stars by Catalano \& Renson (1997). We will discuss only those cases where the period given in this catalogue does not agree (within the error bars) with HVA. In the following we will concentrate on the newly discovered variable $\mathrm{CP}$ stars. The fraction of rotationally variable $\mathrm{CP}$ stars constitutes an increase of about 20 percent related to the catalogue of Catalano \& Renson.

\section{Results}

In order to reinforce the assignments of peculiarity in Renson's catalogue, additional parameters for the intrinsic (chemically) peculiarity such as $\Delta a$ (Maitzen 1976) and $\Delta p$ (Masana et al. 1998) are presented. Unfortunately, $\Delta p$ is not as specific (for CP2 stars) as the $\Delta a$ index especially for stars later than A0. We have not included the Geneva peculiarity index $\Delta(\mathrm{V} 1-\mathrm{G})$ because of its dependence on the interstellar reddening.

Six stars (HD 40312, HD 148112, HD 151965, HD 175362, HD 193722 and HD 219749) from Catalano \& Renson (1997) have a period agreeing with HVA but the period quoted there from the literature does not agree with that of Catalano \& Renson.

Furthermore we have identified eight possible members (HD 4058, HD 75202, HD 79781, HD 95321, HD 111709, 
HD 125081, HD 149420 and HD 167858) of the $\gamma$ Doradus stars (Breger \& Beichbuchner 1996). The given spectral types and found periods are typical for this group of pulsating stars. But further observational efforts are needed to establish their true nature.

The following stars deserve further attention: $H D$ 4058: Budaj (1996) reported a period which is one day longer than that of the HVA.

$H D$ 23848: The period extracted from literature in the HVA is not correct, Martin \& Hube (1988) reported $P=1.765346 \mathrm{~d}$

$H D$ 30466: according to the Photometric Notes and References of HVA the published period 1.3900 days taken from Rakosch \& Fiedler (1978) does not fit the Hipparcos data. Vice versa the Rakosch \& Fiedler data yield a nearly perfect scatter diagram when reduced with the Hipparcos period of 4.0779 days. This fact is supported by the data basis of Maitzen (1977) leading to a period (2.7795 d) not in accord with the HVA period. On the other hand Maitzen's period reveals a double wave light variation in agreement with the light curve of HVA. A possible cause for deriving a discordant period is the low degree of variability in the Hipparcos photometric band whereas the variability for hot CP2 stars is more pronounced in bluer bands where Rakosch \& Fiedler, as well as Maitzen obtained their observations.

HD 7406\%: There seems to be a misclassification of the variability type (BCEP) in the HVA. Since this star is classified as A0 Cr Sr in Renson (1991) we tend to believe that the variations are due to rotation.

HD 8503\%: Renson (1983) derived a period which is half the value from HVA.

$H D$ 126515: There is practically unanimous agreement on a 130 days period of this outstanding magnetic star, based on published photometric, spectroscopic and magnetic field variations (see e.g. North \& Adelman 1995). The HVA period of 3.16414 days yields a light curve with rather enhanced scatter and should be considered as artifact of the intrinsically long period together with a rather low level of variability in the photometric band of Hipparcos.

HD 145792: Cernicharo et al. (1985) used this star as (an a priori constant) comparison star for HD 145102 and HD 147010. They found periods of 1.69253 and $3.99827 \mathrm{~d}$, respectively. These results are probably influenced by the variability of HD 145792 and have to be reanalyzed.

$H D$ 182255: This star is a single-lined spectroscopic binary system with a period of $367 \mathrm{~d}$. Hube \& Aikman (1991) reported additional variations with an interval of $1.09 \mathrm{~d}$. This results is confirmed by HVA although the period is slightly longer.

The newly found rotation periods of stars with known $v \sin i$ values (taken from Uesugi \& Fukuda 1982, Abt \& Morrell 1995 and Levato et al. 1996), were used (34 objects in total) to test the oblique rotator model. An implication of this model is that the relationship between

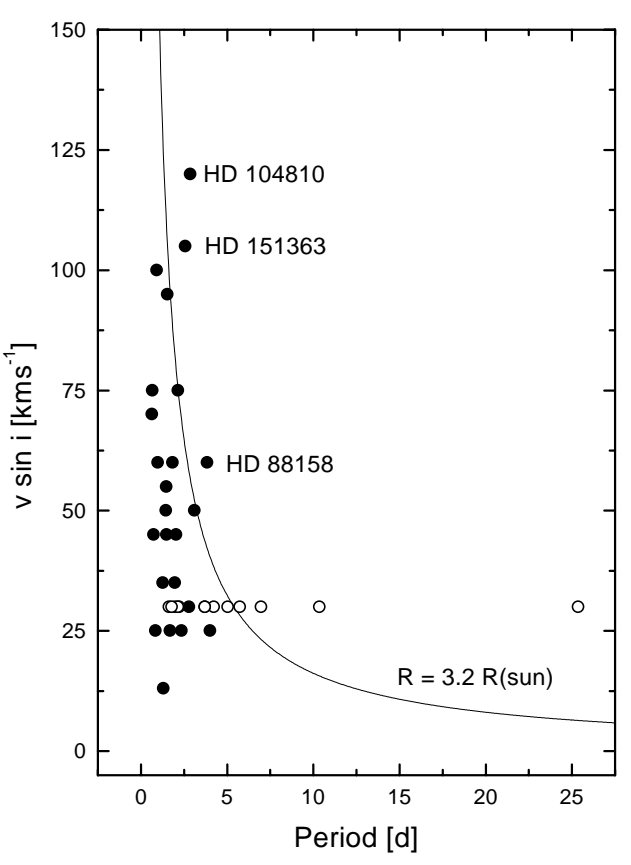

Fig. 1. $P$ vs. $v \sin i$ for $34 \mathrm{CP}$ stars. The open circle indicate stars with only upper values for $v \sin i$ found in the literature

the observed period and the apparent rotation can be estimated as (Preston 1971):

$v=\frac{50.6 R}{P}$

where $v$ is the equatorial rotational velocity, $R$ is the stellar radius in solar units and $P$ is the observed period in days. Since only the projected rotational velocity can be determined, all stars should fall below the given relation assuming a certain stellar radius. Figure 1 shows this relation for the selected 34 objects assuming $R=3.2 R_{\odot}$. Only three stars (HD 88158, B8 Si; HD 104810, B8 Si and HD 151363, B9 Si) seem to lie above the chosen upper limit. Taking into account that in reality there should be a certain spread in stellar radii we feel justified to state that this result fits nicely into the constraints of the oblique rotator model and therefore also supports the peculiar nature of these stars.

It is interesting to note that all 26 bona fide CP1 stars (beside the eight possible $\gamma$ Doradus candidates) are members of eclipsing binary systems and no other kind of variability was detected.

Acknowledgements. This research was carried out within the working group Asteroseismology-AMS with funding from the Fond zur Förderung der wissenschaftlichen Forschung (project S7303-AST). Use was made of the Simbad database, operated at CDS, Strasbourg, France. 


\section{References}

Abt H.A., Morrell N., 1995, ApJS 99, 135

Babcock H.W., 1947, ApJ 105, 105

Breger M., Beichbuchner F., 1996, A\&A 313, 851

Budaj J., 1996, A\&A 313, 523

Budaj J., 1997, A\&A 326, 655

Catalano F.A., Renson P., 1997, A\&AS 121, 57

Cernicharo J., Bachiller R., Duvert G., 1985, A\&A 149, 273

Deutsch A.J., 1970, ApJ 159, 985

Guthnick P., Prager P., 1914, Veröff. K. Sternw. Babelsberg 1,1

Hube D.P., Aikman G.C.L., 1991, PASP 103, 49

Levato H., Malaroda S., Morrell N., Solivella G., Grosso M., 1996, A\&AS 118, 231

Ludendorff H., 1906, Astron. Nach. 173, 1
Maitzen H.M., 1976, A\&A 52, 223

Maitzen H.M., 1977, A\&A 60, L29

Martin B.E., Hube D.P., 1988, IBVS 3240, 1

Masana E., Jordi C., Maitzen H.M., Torra J., 1998, A\&AS 128, 265

North P., Adelman S.J., 1995, A\&AS 111, 41

Preston G.W., 1971, PASP 83, 571

Rakosch K.D., Fiedler W., 1978, A\&AS 31, 83

Renson P., 1983, IBVS 2298, 1

Renson P., 1991, Catalogue Général des Étoiles Ap et Am, Institut d'Astrophysique Université de Liège

Stibbs D.W.N., 1950, MNRAS 110, 395

Uesugi A., Fukuda I., 1982, Revised Catalogue of Stellar Rotational Velocities, Department of Astronomy, Kyoto Univ., Kyoto 